\title{
OPEN Associated Joint Pain With Controlled Ankle Movement Walker Boot Wear
}

Lauren V. Ready, MPH

Erica G. Fisk, MD

William Ciurylo, BA

Christopher P. Chiodo, MD

Eric M. Bluman, MD, PhD

Jeremy T. Smith, MD

From the Department of Orthopaedic Surgery (Ms. Ready), Brigham and Women's Hospital, Brown University; the Department of Orthopaedic Surgery (Dr. Fisk), Brigham and Women's Hospital, OrthoNorCal; the Department of Orthopaedic Surgery (Mr. Ciurylo), Brigham and Women's Hospital, University of New England; and the Department of Orthopaedic Surgery (Dr. Chiodo, Dr. Bluman, and Dr. Smith), Brigham and Women's Hospital, Harvard Medical School, Boston, MA.

Correspondence to Dr. Smith: jsmith42@bwh.harvard.edu

JAAOS Glob Res Rev 2018;2:e044

DOI: 10.5435/

JAAOSGlobal-D-18-00044

Copyright (C) 2018 The Authors. Published by Wolters Kluwer Health, Inc. on behalf of the American Academy of Orthopaedic Surgeons. This is an open-access article distributed under the terms of the Creative Commons Attribution-Non Commercial-No Derivatives License 4.0 (CCBY-NC-ND), where it is permissible to download and share the work provided it is properly cited. The work cannot be changed in any way or used commercially without permission from the journal.

\section{Abstract}

Background: Controlled ankle movement (CAM) walker boots may cause gait alterations and leg-length discrepancy. This study evaluates secondary site pain relating to immobilization in a CAM walker boot.

Methods: Patients wearing a CAM walker boot were prospectively enrolled and evaluated for new or worsened secondary site pain. Surveys at four time points were completed to evaluate secondary site pain severity and its effect on function.

Results: The study included 46 patients (mean age, 49 years). At transition out of the boot (mean, 4.2 weeks), 31 patients (67\%) reported pain which was new or worse than at baseline. The sites most susceptible to pain were lower back, contralateral hip, and ipsilateral knee. Most pains (84\%) began within the first 2 weeks of boot wear. Secondary site pain was less common after transition out of the boot: 18 patients (39\%) at 1 month and 15 patients (33\%) at 3 months.

Conclusion: Secondary site pain after CAM walker boot wear is common. The frequency and severity of pain lessened after transition out of the boot. Yet, one-third of patients still had new or worsened secondary site pain 3 months after cessation of boot wear.

$\mathrm{F}$ Toot and ankle injuries often require management with a controlled ankle movement (CAM) walker boot. ${ }^{1-6}$ CAM walker boots restrict ankle and foot motion and provide a stable platform to distribute forces when weight bearing. Most CAM walker boots are designed to tighten around the extremity and allow the patient to rollover the foot when walking because of the rocker bottom shape of the sole. Thus, CAM walker boots shelter the ankle and foot and can permit a return to ambulation with continued protection of the injured extremity.

Although CAM walker boots are commonly used in the rehabilitation of orthopaedic patients, their use is not entirely benign. Studies have shown altered gait biomechanics created by restricting ankle and foot function. Goodworth et $\mathrm{al}^{7}$ reported increased body motion in patients who undergo range of motion and balance testing when wearing the 
rocker bottom design. Furthermore, the use of a unilateral walking boot creates a simulated leg-length discrepancy, a condition linked to joint pain. ${ }^{7-13}$

To date, little has been published evaluating the association of CAM walker boot wear with pain occurring at a site other than that requiring boot treatment, the so-called secondary site pain. Anecdotally, we have observed many patients reporting secondary site lower back or lower extremity pain when wearing a CAM walker boot. This study evaluates the location, frequency, and duration of secondary site musculoskeletal pain related to immobilization in a CAM walker boot.

\section{Methods}

\section{Patient Population}

Before study initiation, approval was obtained from the Institutional Review Board. One hundred consecutive patients who were placed into a CAM walker boot for management of a foot or ankle injury or disorder were prospectively enrolled in this study. At the time of initiation of boot wear, patients were placed by an orthopaedic cast technician into either a tall Aircast AirSelect Elite or short Aircast AirSelect CAM walker boot, based on the diagnosis and appropriate boot type needed for the treatment. Inclusion criteria included minimum age of 18 years, anticipated boot wear for at least
2 weeks, and weight bearing as tolerated restrictions. Exclusion criteria included transitioning into a CAM walker boot as part of a postoperative protocol, injury requiring restricted weight bearing, or an additional acute injury to the lower back or lower extremity. Those who subsequently reported wearing the boot for less than 2 weeks were excluded. Additionally, patients who had a treatment plan change, such as proceeding with lower extremity surgery during the study period, were removed from the study.

Demographic information was gathered, including age, sex, race or ethnicity, body mass index (BMI), history of lower extremity or lower back injury, history of chronic pain (eg, fibromyalgia, chronic opiate use, chronic regional pain syndrome), and history of cigarette smoking. The diagnosis was recorded for which the CAM walker boot was required.

\section{Outcome Measures}

The primary outcome was secondary site pain, either at lower extremity or lower back, which developed or worsened during CAM walker boot wear. Study participants completed a series of surveys that characterized their lower extremity and lower back pain. Surveys were completed at four time points: initiation of boot wear, transition out of the boot, 1 month after stopping boot wear, and 3 months after stopping boot wear. Patients were asked to document the specific date when the secondary site pain began. Surveys inquired about the presence of secondary site pain, defined as lower back, ipsilateral hip, contralateral hip, ipsilateral knee, contralateral knee, contralateral ankle, and contralateral foot. Severity of pain was assessed using a 100-point visual analog scale (VAS), with zero indicating no pain and 100 representing the worst pain imaginable. ${ }^{14}$ The effect of the secondary site pain on overall function was reported as causing no limitation, some limitation, quite a lot of limitation, and complete limitation. Patient-reported duration and frequency of boot wear were also recorded.

\section{Statistical Analysis}

Patient responses were collected either in a paper form or with use of the Research Electronic Data Capture (REDCap) tool (Vanderbilt University) hosted at our institution. ${ }^{15}$ Statistical analyses were performed using Microsoft Excel (Microsoft Corporation). We used bivariate analysis to assess the relative risk of secondary site pain associated with several covariates. Covariates included sex, age, race, BMI, injury type, history of lower extremity/back injury, chronic pain, type of CAM walker boot (tall/short), and duration of boot wear. Age was categorized as younger than or equal to 30 years, 31 to 59 years, and 60 years and older. Because of a predominantly Caucasian population, our race analysis was separated into Caucasian, not Caucasian, and unknown. BMI was separated into normal at under 25 ,

Ready or an immediate family member serves as a paid consultant to Smith \& Nephew and Stryker. Dr. Fisk or an immediate family member serves as a paid consultant to Synthes. Dr. Chiodo or an immediate family member has received IP royalties from Aircast(DJ), Arthrex, Darco, and Zimmer Biomet; has stock or stock options held in Johnson \& Johnson, Merck, and Zimmer Biomet; and serves as a board member, owner, officer, or committee member of the American Academy of Orthopaedic Surgeons, American Orthopaedic Foot and Ankle Society, and Mass Ortho Association. Dr. Bluman or an immediate family member has stock or stock options held in EDC; has received nonincome support (such as equipment or services), commercially derived honoraria, or other non-research-related funding (such as paid travel) from Arthrex and Rogerson Orthopaedics; and serves as a board member, owner, officer, or committee member of the American Orthopaedic Foot and Ankle Society. Dr. Smith or an immediate family member serves as a board member, owner, officer, or committee member of the American Orthopaedic Foot and Ankle Society. Neither Mr. Ciurylo nor any immediate family member has received anything of value from or has stock or stock options held in a commercial company or institution related directly or indirectly to the subject of this article. 
overweight between 25 and 30, and obese if above 30 . The diagnosis possibilities were sprain, fracture, tendon problem, or other, and the duration of boot wear ranged from 2 to 4 weeks, 4 to 6 weeks, and more than 6 weeks. Chronic pain was identified through medical record review as chronic pain, fibromyalgia, complex regional pain syndrome, or reflex sympathetic dystrophy. We used a modified Poisson regression with robust error variance to estimate the relative risk. This approach was used rather than logistic regression, because the odds ratio can overstate the risk in the case of common outcomes. ${ }^{16} P$ value less than 0.05 was considered statistically significant. All analyses were conducted using SAS software version 9.4 (SAS Institute).

\section{Results}

One hundred patients were invited to participate in this study. Of these, 3 declined initial participation, 36 did not complete all surveys, and 15 were excluded because they did not wear the CAM walker boot for 2 weeks or had a change in treatment. The final study population included $46 \mathrm{pa}$ tients, of whom 15 were men $(33 \%)$ and 31 women $(67 \%)$ with a mean age of 49 years (range, 22 to 77 years). Most patients (85\%) were Caucasian (Table 1).

The diagnosis requiring CAM walker boot treatment was a fracture in 17 patients (37\%), a sprain in 7 patients $(15 \%)$, and a tendon disorder in 17 patients $(37 \%)$. Five patients $(11 \%)$ were treated with a boot for a gastrocnemius strain, stress reaction, plantar plate injury, or plantar fascia rupture (Table 1). Thirty-one patients were treated with a tall CAM boot and 15 with a short boot. Mean duration of boot wear was 4.2 weeks (range, 2 to 9 weeks), wearing the boot for a

Table 1

\section{Patient Demographics}

\begin{tabular}{lc}
\hline Demographic & Number \\
\hline Age (yr, mean, range) & $49.8(22-77)$ \\
Sex (n, \%) & \\
Male & $15(33)$ \\
Female & $31(67)$ \\
Smoker (n, \%) & \\
Yes & $1(2)$ \\
No & $45(98)$ \\
Race/ethnicity (n, \%) & $39(85)$ \\
Caucasian & $2(4)$ \\
Hispanic & $1(2)$ \\
Asian & $2(4)$ \\
Black/African American & $2(4)$ \\
Declined & \\
Injury (n, \%) & $17(37)$ \\
Fracture & $7(15)$ \\
Sprain & $17(37)$ \\
Tendon problem & $5(11)$ \\
Other & \\
Body mass index (kg/m $\left.{ }^{2}, \mathrm{n}, \%\right)$ & $24(52)$ \\
Normal (<25) & $10(22)$ \\
Overweight (25-30) & $12(26)$ \\
Obese (>30) & \\
\hline
\end{tabular}

${ }^{a}$ Gastrocnemius strain, stress reaction, plantar plate injury, or plantar fascia rupture.

mean of 6.5 (range, 3 to 7 ) days per week for an average of 11.6 (range, 3 to 23) hours per day.

At the time of initiation of boot wear, 27 patients $(59 \%)$ reported one or more secondary site pains. Among these 27 patients, a total of $50 \mathrm{sec}-$ ondary site pains were reported. The most frequent baseline pain sites were the lower back, ipsilateral knee, and contralateral hip. At baseline, the mean VAS of secondary site pain was 53.1 (range, 3 to 97).

At the time of transition out of the boot, 31 patients $(67 \%)$ reported secondary site pain which was either new or worse than at baseline. Of these, 13 reported pain at a new secondary site and 18 reported worsening of baseline secondary site pain. Seventeen of these patients experienced new or worsening pain in the lower back, 4 the ipsilateral hip, 11 the contralateral hip, 10 the ipsilateral knee, 8 the contralateral knee, 2 the contralateral ankle, and 1 the contralateral foot (Figure 1). Of those with new or worsened secondary site pain, each patient experienced an average of 1.6 secondary sites of pain at the time of transitioning out of the boot.

One month after transitioning out of the boot, 18 patients $(39 \%)$ reported persistent secondary site pain which had developed during boot wear and was either new or worse than at baseline. Three months after transitioning out of the boot, 15 patients $(33 \%)$ reported persistent secondary site pain. Of these, 12 had reported secondary site pain at baseline. Thus, $48 \%$ of those in whom secondary site pain developed while 


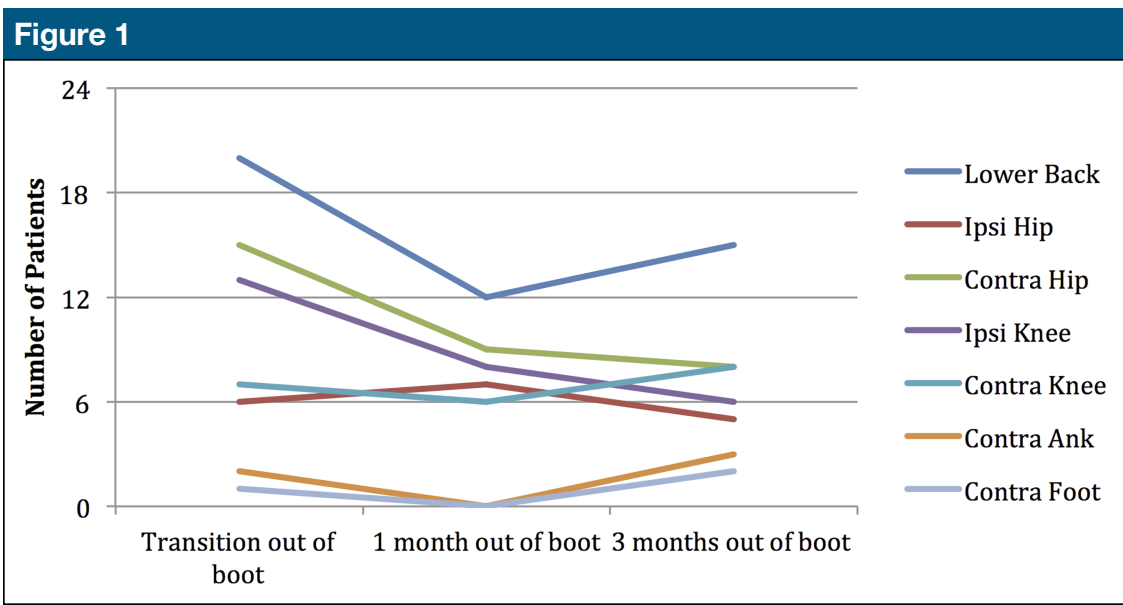

Number of patients reporting new or worsened secondary site pain at each time point. Specific sites of pain are represented by colors.

wearing the boot still had secondary site pain 3 months after cessation of boot wear.

Most $(84 \%)$ of the new or worsened secondary site pains began within the first 2 weeks of boot wear. Of patients' secondary site pains, 34 sites caused little to no limitation, 21 some limitation, 10 quite a lot of limitation, and 4 complete limitation of the patient's usual activities. Mean VAS for secondary site pains at the time of transition out of the boot was 51.2 (range, 4 to 98). One month after coming out of the boot, mean VAS was 57.4 (range, 16 to 100 ) and at 3 months after boot wear, mean VAS was 43.2 (range, 15 to 99 ).

In 22 patients with a history of lower back or lower extremity pain, new or worsened secondary site pain developed in 16 of them during boot wear. Secondary site pain developed in all enrolled patients with a history of chronic pain (8/8) and in one patient who smoked cigarettes. No statistically significant correlation exists between the development of secondary site pain and sex, age, race or ethnicity, BMI, diagnosis, history of lower back or lower extremity injury, smoking, use of tall or short boot, or duration of boot wear. However, statistical significance was found correlating secondary site pain with a history of chronic pain (Table 2).

\section{Discussion}

The severity and frequency of secondary site pain related to immobilization in a CAM walker boot are yet to be evaluated in the literature. In this study, $67 \%$ of patients treated with a CAM walker boot for at least 2 weeks reported new or worsened secondary site pain at the time of transition out of the boot. On average, each of these patients experienced 1.6 secondary pain sites. The reasons for secondary site pain during CAM walker boot wear are likely multifactorial and could be related to boot design, altered gait mechanics, or simulated leg-length discrepancy.

Most CAM walker boots have a rocker bottom sole, which is designed to facilitate roll-through during ambulation. The advantage of this is to shield the ankle and foot during the gait cycle. Yet, this can lead to alterations in balance and an altered postural response during gait. ${ }^{17-19}$ Furthermore, decreasing ankle range of motion diminishes proprioceptive feedback through the lower extrem- ity, resulting in abnormal kinematics at other more proximal joints. ${ }^{7}$

CAM walker boots also create a simulated leg-length discrepancy. ${ }^{7}$ Leg-length inequality can cause abnormal joint forces and motion in the lower extremity. Physiologic compensation to leg-length discrepancy occurs, including bending of the longer-leg knee, which adds pressure to the lateral patellofemoral articulation. ${ }^{20}$ Additionally, the pelvis can tip and become lower on the shorter side, decreasing the femoral head coverage on the longer side. ${ }^{10}$ The short-sided foot externally rotates, repositioning the heel into valgus and collapsing the longitudinal arch. ${ }^{12}$ Rotation of the longer limb has also been implicated in progressive scoliotic curvature, which may lead to development of lower back pain. ${ }^{21-28}$ Betsch et $\mathrm{al}^{9}$ illustrated that an artificial leg-length inequality of just $15 \mathrm{~mm}$ can lead to changes in pelvic tilt, torsion, and spinal posture. Long-term effects of limb-length inequality include the development of arthritis of the hip and lower back pain. ${ }^{29}$

We can draw several conclusions about the timing and severity of pain onset related to CAM walker boot wear. Most $(84 \%)$ of the new or worsened secondary site pains began within the first 2 weeks of wearing the boot. And, although it did not reach statistical significance, we observed a trend of those spending a longer time in the boot having more frequent and severe pain. With respect to recovery after transitioning out of the boot, $52 \%$ of patients' new or worsened secondary site pain completely resolved by 3 months after ceasing boot wear. The mean severity of pain, as measured by patient-reported VAS, initially worsened after ceasing boot wear and then demonstrated a trend of improvement by 3 months. Taken together, these findings suggest a dose-related effect of boot wear, with longer use correlated to more 
Table 2

\section{Factors Associated With Secondary Site Pain}

\begin{tabular}{|c|c|c|c|}
\hline \multirow[b]{2}{*}{ Variable } & \multirow[b]{2}{*}{ Presence of Secondary Site Pain (\%, Number) } & \multicolumn{2}{|c|}{ Univariate } \\
\hline & & RR $(95 \%$ Cl) & $P$ Value \\
\hline Sex & & & 0.51 \\
\hline Male $^{\mathrm{a}}$ & $60 \%(9 / 15)$ & 1.00 & \\
\hline Female & $71.0 \%(22 / 31)$ & $1.18(0.74-1.89)$ & \\
\hline Age & & & 0.38 \\
\hline 30 years or younger ${ }^{a}$ & $57.1 \%(4 / 7)$ & 1.00 & \\
\hline $31-59$ years & $76.0 \%(19 / 25)$ & $1.33(0.67-2.62)$ & \\
\hline 60 years or older & $57.1 \%(8 / 14)$ & $1.00(0.46-2.19)$ & \\
\hline Caucasian & & & 0.68 \\
\hline $\mathrm{No}^{\mathrm{a}}$ & $60.0 \%(3 / 5)$ & 1.00 & \\
\hline Yes & $69.2 \%(27 / 39)$ & $1.15(0.55-2.43)$ & \\
\hline Unknown & $50.0 \%(1 / 2)$ & $0.83(0.18-3.96)$ & \\
\hline Body mass index $\left(\mathrm{kg} / \mathrm{m}^{2}\right)$ & & & 0.32 \\
\hline Normal $(<25)^{\mathrm{a}}$ & $75.0 \%(18 / 24)$ & 1.00 & \\
\hline Overweight (25-30) & $70.0 \%(7 / 10)$ & $0.93(0.59-1.49)$ & \\
\hline Obese $(>30)$ & $50.0 \%(6 / 12)$ & $0.67(0.36-1.23)$ & \\
\hline Diagnosis & & & 1.00 \\
\hline Sprain $^{\mathrm{a}}$ & $71.4 \%(5 / 7)$ & 1.00 & \\
\hline Fracture & $70.6 \%(12 / 17)$ & $0.99(0.56-1.73)$ & \\
\hline Tendon problem & $64.7 \%(11 / 17)$ & $0.91(0.50-1.63)$ & \\
\hline Other & $60.0 \%(3 / 5)$ & $0.84(0.36-1.98)$ & \\
\hline Previous lower back/extremity injury & & & 0.54 \\
\hline $\mathrm{No}^{\mathrm{a}}$ & $62.5 \%(15 / 24)$ & 1.00 & \\
\hline Yes & $72.7 \%(16 / 22)$ & $1.16(0.78-1.74)$ & \\
\hline Chronic pain & & & 0.04 \\
\hline $\mathrm{No}^{\mathrm{a}}$ & $60.5 \%(23 / 38)$ & 1.00 & \\
\hline Yes & $100 \%(8 / 8)$ & $1.65(1.28-2.14)$ & \\
\hline Boot height & & & 0.51 \\
\hline Tall $^{\mathrm{a}}$ & $71.0 \%(22 / 31)$ & 1.00 & \\
\hline Short & $60.0 \%(9 / 15)$ & $0.85(0.53-1.35)$ & \\
\hline Duration of boot wear & & & 0.52 \\
\hline $2-4 w^{a}$ & $61.3 \%(19 / 31)$ & 1.00 & \\
\hline $4-6 w k$ & $75.0 \%(9 / 12)$ & $1.22(0.80-1.88)$ & \\
\hline$>6 w k$ & $100.0 \%(3 / 3)$ & $1.63(1.23-2.16)$ & \\
\hline
\end{tabular}

frequent secondary site symptoms and a gradual subsequent recovery after the patient no longer requires the boot.

In this study, patients with a history of chronic pain were at particular risk for development of secondary site pain. Although we additionally noted an increased relative risk of secondary site pain in other subgroups, these analyses did not reach statistical significance. Patients with a history of chronic pain who require CAM walker boot treatment might warrant additional counseling about the risks of pain related to boot wear.
In this study, the lower back was the most common secondary pain site, followed by the contralateral hip and ipsilateral knee. These sites were also the most common baseline secondary pain sites. These data suggest that although secondary site pain can develop at new sites while wearing a 
CAM walker boot, preexisting secondary pain sites are particularly likely to be exacerbated. The contralateral ankle and foot were not frequent secondary pain sites.

Limitations of this study include its small sample size and that only $46 \%$ of patients who met inclusion criteria completing all surveys. It is possible that those responding to the surveys in full were more, or less, likely than the general population to have secondary site pain. The patientreported nature of the surveys also generates a potential response bias, although the subjective nature of pain necessitates a study design that is based on patient reporting. Another limitation is the wide range of hours per day that the CAM walker boot was worn by patients. It is possible that patients may have modified the duration of boot wear to minimize secondary site pain. If this was the case, it would likely have the effect of diminishing the severity of reported secondary site pain. We did not address this possible occurrence in our surveys or the possibility that foot shape and alignment contributed to the boot experience. Additionally, we cannot be certain that the boot wear was causative of the secondary site pain, because patients in this study did have a lower extremity injury or disorder. It is plausible that the altered gait contributing to the secondary site pain may have been because of the injury more than the boot itself.

Additional research into the relationship between CAM walker boot wear and secondary site pain may further define the effects of the boot as opposed to the injury. It would be beneficial to further evaluate the leglength discrepancy and variation in gait experienced by patients wearing a CAM walker boot. Wearing a heel or a shoe lift on the contralateral extremity would theoretically eliminate the leg-length discrepancy and may minimize the frequency of sec- ondary site pain. It would also be of interest to evaluate the secondary site pain at 6 months and a year to determine if the symptoms resolved.

Given the frequency with which CAM walker boots are prescribed, it is important to understand the association between boot wear and secondary site pain. In our practice, patients frequently inquire about whether a CAM walker boot will cause or exacerbate pain. Because of the results of this study, we are now better able to counsel patients and are more likely to recommend devices that balance the leg lengths when wearing a boot or use crutches to assist with pain.

\section{Conclusion}

Sixty-seven percent of patients included in this study experienced new or worsened secondary site pain during CAM walker boot wear for a lower extremity injury or disorder. The sites most susceptible to secondary pain were the lower back, contralateral hip, and ipsilateral knee. Although the frequency and severity of secondary site pain improved with time after transitioning out of the boot, one-third of patients had persistent secondary site pain 3 months after cessation of boot wear.

\section{Acknowledgments}

The authors thank Jamie E. Collins, $\mathrm{PhD}$, for help with the statistical analysis.

\section{References}

1. DiGiovanni BF, Moore AM, Zlotnicki JP, Pinney SJ: Preferred management of recalcitrant plantar fasciitis among orthopaedic foot and ankle surgeons. Foot Ankle Int 2012;33:507-512.

2. Hsu RY, Bariteau J: Management of ankle fractures. R I Med J 2013;96:23-27.

3. Hubbard TJ, Wikstrom EA: Ankle sprain: Pathophysiology, predisposing factors, and management strategies. Open Access J Sports Med 2010;1:115.

4. Hunt KJ, Goeb Y, Esparza R, Malone M, Shultz R, Matheson G: Site-specific loading at the fifth metatarsal base in rehabilitative devices: Implications for Jones fracture treatment. Am J Phys Med Rehabil 2014;6: 1022-1029.

5. Simpson MR, Howard TM: Tendinopathies of the foot and ankle. Am Fam Physician 2009;80:1107-1114.

6. Vulcano E, Deland JT, Ellis SJ: Approach and treatment of the adult acquired flatfoot deformity. Curr Rev Musculoskelet Med 2013;6:294-303.

7. Goodworth AD, Kunsman M, DePietro V, LaPenta G, Miles K, Murphy J: Characterization of how a walking boot affects balance. J Prosthet Orthot 2014;26: 54-60.

8. Walker Boot. (New Products). Surgical Products June 2003: 22. Academic OneFile. Web. 18 May 2016.

9. Betsch M, Wild M, Große B, Rapp W, Horstmann T: The effect of simulating leg length inequality on spinal posture and pelvic position: A dynamic rasterstereographic analysis. Eur Spine J 2012;21:691-697.

10. Bhave A, Paley D, Herzenberg JE: Improvement in gait parameters after lengthening for the treatment of limb-length discrepancy. J Bone Joint Surg 1999;81: 529-534.

11. Golightly Y, Allen K, Helmick C, Renner J, Jordan J: Symptoms of the knee and hip in individuals with and without limb length inequality. Osteoarthritis Cartilage 2009; 17:596-600.

12. McCaw S, Bates B: Biomechanical implications of mild leg length inequality. Br J Sports Med 1991;25:10-13.

13. Wretenberg P, Hugo A, Broström E: Hip joint load in relation to leg length discrepancy. Med Devices (Auckl) 2008;1:13-18.

14. Hawker GA, Mian S, Kendzerska T, French M: Measures of adult pain: Visual analog scale for pain (VAS pain), numeric rating scale for pain (NRS pain), mcgill pain questionnaire (MPQ), short-form mcgill pain questionnaire (SF-MPQ), chronic pain grade scale (CPGS), short form-36 bodily pain scale (SF-36 BPS), and measure of intermittent and constant osteoarthritis pain (ICOAP). Arthritis Care Res 2011; 63(suppl 11):S240-S252.

15. Harris PA, Taylor R, Thielke R, Payne J, Gonzalez N, Conde JG: Research electronic data capture (REDCap)-A metadata-driven methodology and workflow process for providing translational research informatics support. J Biomed Inform 2009;42:377-381.

16. Zou G: A modified Poisson regression approach to prospective studies with binary data. Am J Epidemiol 2004;159:702-706. 
17. Albright BC, Woodhull-Smith WM: Rocker bottom soles alter the postural response to backward translation during stance. Gait Posture 2009;30:45-49.

18. Horak FB, Nashner LM: Central programming of postural movements: Adaptation to altered support-surface configurations. J Neurophysiol 1986;55: 1369-1381.

19. van Deursen R: Footwear for the neuropathic patient: Offloading and stability. Diabetes Metab Res Rev 2008;24(suppl 1):S96-S100.

20. Greenfield B. Evaluation of overuse syndromes. In: Donatelli R (ed.), Biomechanics of the Foot and Ankle. New York, NY: FA Davis, 1990;153-177.
21. Blake R, Ferguson H: Limb length discrepancies. J Am Podiatr Med Assoc 1992;82:33-38.

22. Blustein SM, D'Amico JC: Limb length discrepancy: Identification, clinical significance, and management. $J A m$ Podiatr Med Assoc 1985;75:200-206.

23. Friberg O: Clinical symptoms and biomechanics of lumbar spine and hip joint in leg length inequality. Spine 1983;8:643-651.

24. Gofton J: Persistent low back pain and leg length disparity. J Rheumatol 1985;12: 747-750.

25. Langer S: Structural leg shortage: A case report. J Am Podiatr Med Assoc 1976;66:38-40.
26. McCaw ST: Leg length inequality. Implications for running injury prevention. Sports Med 1992;14:422-429.

27. Subotnick SI: Limb length discrepancies of the lower extremity (the short leg syndrome). J Orthop Sports Phys Ther 1981;3:11-16.

28. Woerman AL, Binder-Macleod SA: Leg length discrepancy assessment: Accuracy and precision in five clinical methods of evaluation. J Orthop Sports Phys Ther 1984;5:230-239.

29. Steen H, Terjesen T, Bjerkreim I: Anisomelia: Clinical consequences and treatment [in Norwegian]. Tidsskr Nor Laegeforening 1997;117:1595-1600. 